\title{
Four Weeks of 16:8 Time-Restricted Feeding on Stress, Sleep, Quality of Life, Hunger Level, and Body Composition in Healthy Adults: A Pilot Study on Wellness Optimization
}

\author{
Gurinder Bains, $\mathrm{PhD}^{1 *}$, Everett Lohman, DSc, PT, OCS ${ }^{1}$, Michael Moh, MS, RD ${ }^{1}$, Noha Daher, DrPH ${ }^{1}$, \\ Lee Berk, DrPH, MPH, FACSM, CHES, CLS'
}

\begin{abstract}
Introduction: Sustainable methods of dietary lifestyle changes are essential to long term weight loss maintenance and enhancement in overall health and wellness. A novel modified alternative method to caloric restriction is Time-Restricted Feeding (TRF), a sub-type of Intermittent Fasting. The purpose of this pilot study was to determine the effect of four weeks of TRF (without caloric deficit) on body composition, stress levels, sleep quality, hunger level, and quality of life.
\end{abstract}

Methods: Sixteen participants, 9 males and 7 females, mean age $34.0 \pm 11.7$ years, completed the four-week protocol. Fasting program was 16 hours from the time of last meal of the evening to start of first meal the following day. Participants were required to consume their normal daily caloric intake within an 8-hour period.

Results: There were significant reductions in mean body fat percentage $(26.2 \pm 2.4$ vs. $25.5 \pm 2.6, \mathrm{p}=0.01)$, visceral fat $(\mathrm{cm} 2)(98.2 \pm 15.3$ vs. $94.1 \pm 15.7, \mathrm{p}=0.003)$, body fat mass $(\mathrm{kg})(21.6 \pm 3.0 \mathrm{vs.} 21.0 \pm 3.2, \mathrm{p}=0.02)$, trunk fat $(\mathrm{kg})(11.5 \pm 1.6$ vs. $11.0 \pm 1.6, \mathrm{p}=0.001)$ and stress levels $(14.1 \pm 1.9$ vs. $11.0 \pm 1.4, \mathrm{p}=0.03)$. There was a significant drop in hunger levels over time $(\chi 2=16.4, \mathrm{p}=0.001)$. There were no significant changes in sleep quality or quality of life.

Conclusion: TRF may be an effective option to decrease stress, body fat, visceral fat, and obesity-related diseases while not disrupting sleep quality and Quality of Life. Our pilot study demonstrated potential health benefits that could be incorporated by healthcare workers into whole-person wellness programs.
DOI: 10.18297/jwellness/vol3/ iss $2 / 10$

Received Date: July 13, 2021 Accepted Date: Oct 26, 2021 Publication Date: Nov 12, 2021 Website: https://ir.library.louisville.edu/jwellness/

Recommended Citation: Bains, Gurinder; Lohman, Everett; Moh, Michael; Daher, Noha; and Berk, Lee (2021) "Four Weeks of 16:8 Intermittent Fasting on Stress, Sleep, Quality of Life, and Body Composition in Healthy Adults: A Pilot Study on Wellness," Journal of Wellness: Vol. 3 : Iss. 2 , Article 10.

Affiliations: ${ }^{1}$ Loma Linda University

\section{INTRODUCTION}

According to the World Health Organization, obesity rates worldwide have practically tripled since 1975 [1]. According to the Center for Disease Control National Center for Health Statistics, the age-adjusted prevalence of obesity was approximately $42 \%$ in adults in the United States during 2017-2018 [2]. Weight loss and improvement in body composition through physical activity and/or dietary modifications may help decrease the risk for obesity-related disease [3]. The International Food Information Council (IFIC) Foundation's Annual Survey (2018) indicated that $36 \%$ of Americans followed a particular eating plan or diet [4]. The eating design revealed to be most utilized was Intermittent Fasting (IF) [4].

Weight management strategies are numerous and focus primarily on physical activity and/or diet regimens in lieu of lifestyle changes $[5,6]$. There are many distinct diet paradigms: such as those emphasizing consumption or an increase/decrease in protein, carbohydrates, or fat, tracking daily caloric intake, and caloric restriction $[5,7,8]$. Utilizing traditional continuous energy or caloric restriction as the driving force to lose weight can be challenging to sustain weight loss $[9,10]$. Sustainable methods of dietary lifestyle changes are essential to long term weight loss maintenance and enhancement in overall health and wellness $[11,12]$. One such promising approach is IF.

IF, also known as intermittent caloric or energy restriction is a novel alternative method for caloric restriction. IF is a hypernym for a variety of voluntary time restricted eating schedules for weight management. The focal point of IF is based on the explicit time duration of fasting and eating. IF can be further categorized into 3 sub-classifications: 1) Alternate-day fasting (e.g. 5:2 diet), 2) periodic fasting, and 3) daily Time-Restricted Feeding (TRF) $[5,13,14]$. IF (time restricted feeding) has become well-known and popular through the last few years. TRF entails consuming calories during a specified number of consecutive hours (eating window) during a day without any alterations to normal daily caloric intake [15]. Common eating windows are between 4-10 hours [16]. One prevailing method of TRF is the 16:8 approach, also known as 16:8 IF. Ad libitum calories, eating as much or as often as desired, are consumed during an eight-hour period and fasting begins immediately afterward for 16 hours [16]. During the fasting period, water and non-caloric beverages are permitted [5]. 
A substantial amount of human research on the health benefits of IF has emerged. IF may improve body composition, decrease body weight, improve glucose tolerance, improve lipid profile, decrease blood pressure, decrease risk of cancer, and may be neuroprotective and cardioprotective [15, 17-22].

Tracking daily calories can be a component of weight loss; however, tracking calories can become tedious, with adherence to tracking calories demonstrating a decline over time [23, 24]. Similar to recent studies in IF and TRF [16, 25-30], this intervention paradigm was designed to have an ease of implementation that would be attractive to busy individuals where tracking of calories would not be required, thus hopefully decreasing the dietary intervention failure rate. Relatively new in the literature [16], this 16:8 TRF protocol would carry with it no prescribed self-reported caloric deficit, while also requiring no tracking of daily calorie consumption.

Although similar trials have evaluated the $16: 8$ protocol [25-29], to the best of our knowledge there have been no studies that have assessed 16:8 TRF (with no prescribed caloric deficit) on cumulative wellness measures: acute stress, quality of life, hunger level, body composition, and sleep quality in healthy individuals. Therefore, the purpose of this pilot study was to determine the effect of four weeks of 16:8 TRF, with a daily fasting window of 16 hours, requiring no self-reported caloric deficit, on acute stress levels, sleep quality, quality of life, level of hunger, and body composition in healthy individuals.

\section{METHODS}

\section{Participants}

Participants recruited for this prospective pilot study were healthy individuals between the ages of 18 to 65 years who have never attempted a 16:8 IF protocol. Potential participants were excluded if they were diagnosed with diabetes or the risk factors associated with Metabolic Syndrome. Loma Linda University Institutional Review Board approved all methods and procedures (IRB reference 5170221).

Potential participants were recruited at Loma Linda University, Loma Linda, California, and surrounding communities. All participants except one were Loma Linda University students, faculty, and staff. Recruitment was performed through flyers that were posted and emailed at Loma Linda University, and word of mouth by research investigators on campus.

\section{Study Design}

The purpose and procedures of the study were explained to potential participants and all questions were satisfactorily answered. If individuals were interested in participating in the study, a day and time was set where Informed Consent was signed. Baseline assessments of height, body composition (InBody 770), and questionnaire on sleep (Pittsburgh Sleep Quality Index) and stress (Perceived Stress Scale) were completed. A daily compliance log sheet was explained and administered. All measurements were re-evaluated at week four. There was no control group in this pilot study.

\section{Intervention}

The IF protocol was set for a 4-week period using the subtype of IF known as TRF, comprising a daily fasting protocol of 16 hours, which was unrestricted to the time of day. The participants were required to fast 16 hours from the time of their last meal in the evening, through sleep, until the start of their first meal the following day. During the fasting period, the participants were permitted to drink water, black coffee/tea, or zero calorie drinks. Once the fast was broken, the participant was instructed to "eat the same amount of food you normally would in a day" within an 8-hour period. Other protocols have evaluated 8-hour eating periods that were required to start or end at certain times of day [5], however this intervention was not restricted in this manner. Also, there was no official tracking of daily calories using an app or log sheet. There was no requirement on the number of separate meals consumed during the eight hours. Compliance was measured using a 28-day log sheet. Participants recorded a yes / no on the daily compliance log sheet for the 28 days. Participants were contacted throughout the study to verify any unforeseen circumstances preventing IF.

\section{Outcomes}

The outcome measures (body composition, and subject reported outcomes) were assessed at baseline and at four weeks. Height was measured using the InBody digital free-standing Biospace Stadiometer (BSM170). Participants stood erect and barefoot for accurate measurement. Body Composition was performed using the InBody 770 (Cerritos, CA). Excellent agreements were observed between dual-energy $\mathrm{x}$-ray absorptiometry scan and InBody when determining whole body lean mass and percentage body fat composition (Intraclass correlation coefficient ranged from 0.93 to 0.99 ) in overweight and normal individuals [31]. The output report was comprised of three domains: Body Composition and Analysis, Obesity Diagnosis, and Segmental Lean Development. Body Composition and Analysis includes measurements on weight, skeletal muscle mass, dry lean mass, body fat mass and intracellular and extracellular water. Obesity Diagnosis consists of Body Mass Index and Percent Body Fat. Segmental Lean Development assesses the right and left arm, the trunk, and the right and left leg.

\section{Subject Reported Outcomes}

Stress:

The Perceived Stress Scale by Cohen (PSS-10) was administered to determine participant awareness to their level of stress over four weeks [32]. Questions concentrated on feelings and thoughts. Scores above 20 indicate high stress levels while a score of about 6 is average. The PSS-10 demonstrates adequate validity, test-retest reliability, and internal consistency among various populations [33].

Sleep:

The Pittsburgh Sleep Quality Index (PSQI) was used to measure the quality and patterns of sleep over four weeks. The seven elements of the index are sleep latency, subjective sleep quality, 
sleep duration, sleep disturbances, habitual sleep efficiency, use of sleeping medications, and daytime dysfunction. A total score of $\geq 5$ implies poor sleep quality. The PSQI has adequate content validity and adequate internal consistency [34].

\section{Quality of life:}

The 36-Item Short Form Survey Instrument (SF-36) is a valid tool to measure certain aspects of Quality of Life [35]. The SF-36 is comprised of eight disciplines: physical functioning, role limitation due to physical health, role limitations due to emotional problems, energy/fatigue, emotional well-being, social functioning, general health, and pain. Responses were for the previous 4 weeks.

\section{Hunger:}

A Visual Analog Scale (VAS) was administered to measure the level of subjective hunger over four weeks. A hunger VAS shows good within-subject reliability and validity [36]. Participants marked their level of hunger on the $100 \mathrm{~mm}$ VAS line with the low end representing "not hungry at all" and the high end representing "extremely hungry".

\section{Activity:}

Self-reported activity Level was based upon the 5-point scale employed in the Harris Benedict Basal Metabolic Rate equation: sedentary $=1$, lightly active $=2$, moderately active $=3$, very active $=4$, and extra active $=5$.

\section{Statistical Methods}

Data were summarized using mean and standard deviation (SD) for quantitative variables and counts (\%) for qualitative variables. The normality of continuous variables was examined using Shapiro Wilk's test and Boxplots. A paired t-test was performed to determine if there were changes in the outcome measures over time (baseline versus 4 weeks) following the IF program. Wilcoxon signed rank test was used to examine changes in SF-36 scores overtime. Friedman test was used to examine the changes in median VAS hunger scores over 4 periods of time (baseline, week 2, week 3, and week4). Wilcoxon signed rank test was used to examine post-hoc changes in VAS hunger scores over the different periods of time. All statistical analyses were performed using IBM SPSS Statistics Software version 24 for Windows (Chicago, IL, USA) and level of significance was set at $\mathrm{p} \leq 0.05$.

\section{RESULTS}

A cohort of 16 subjects with mean age of $34.0 \pm 11.7$ years and body mass index (BMI) $27.4 \pm 1.3 \mathrm{~kg} / \mathrm{m} 2$ were recruited for this pilot study. A flowchart of participation is illustrated in Figure 1. Most of the participants were males $(n=9,56.3 \%)$ and reported moderate activity level $(n=15,93.8 \%)$. The self-reported mean compliance (via daily journal) of following the rigorous IF program was $24.2 \pm 4.0$ days out of a possible 28 days. Fifteen participants were day shift workers. Eleven participants were graduate students, four worked in healthcare, and one worked as a mechanic. Participant demographics are illustrated in Table 1.

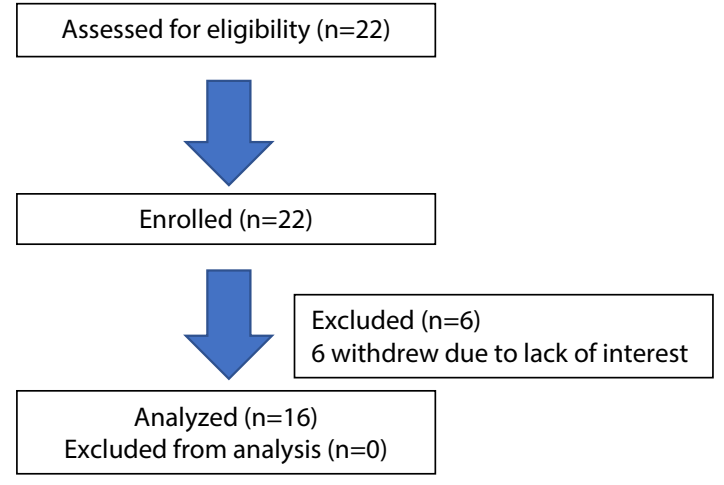

Figure 1. Flow of participants assessed, enrolled, and included in analysis

Changes in stress, body fat composition, and sleep quality across baseline versus four weeks are displayed in Table 2 (next page). There was a significant reduction in stress (mean \pm standard error) ( $14.1 \pm 1.9$ vs. $11.0 \pm 1.4, \mathrm{p}=0.03)$, body fat $\%(26.2 \pm 2.4$ vs. $25.5 \pm 2.6, \mathrm{p}=0.01)$, body fat mass $(\mathrm{kg})(21.6 \pm 3.0$ vs. $21.0 \pm 3.2$, $\mathrm{p}=0.02)$, visceral fat $(\mathrm{kg})(98.2 \pm 15.3$ vs. $94.1 \pm 15.7, \mathrm{p}=0.003)$, and trunk fat mass $(\mathrm{kg})(11.5 \pm 1.6$ vs. $11.0 \pm 1.6, \mathrm{p}=0.001)$ (Table 2$)$. A large effect size was seen in the change in trunk and visceral fat $(\mathrm{d}=1.10$ and 0.81 , respectively). A medium effect size was seen in changes in stress, body fat \%, and body fat mass $(\mathrm{d}=0.50,0.68$, and 0.58 , respectively). Although there was a trend towards statistical significance in BMI ( $\mathrm{p}=0.06)$, a medium effect size was seen $(d=0.50)$. There was no significant change in sleep quality, weight, BMI, skeletal muscle mass, and lean body mass ( $\mathrm{p}>0.05$, Table 2).

Changes in median (min, max) of VAS hunger scores over time are shown in Figure 2 (next page). There was a significant drop in hunger scores over time $\left(\chi^{2}=16.4, \mathrm{p}=0.001\right)$. There was a significant change in median (minimum, maximum) VAS score between baseline and week $3((71.4(0,100)$ versus $(43.6$ $(0,90), \mathrm{Z}=-2.27, \mathrm{p}=0.023)$; baseline and week $4((71.4(0,100)$ versus $(31.5(0,91), Z=-2.27, \mathrm{p}=0.023)$; week 2 and week 3 ((55.8 $(0,95)$ versus $(43.6(0,90), \mathrm{Z}=-2.16, \mathrm{p}=0.031)$; week 2 and week $4((55.8(0,95)$ versus $(31.5(0,91), \mathrm{Z}=-2.39, \mathrm{p}=0.017)$; and week 3 and week $4((43.6(0,90)$ versus $(31.5(0,91), \mathrm{Z}=-2.5, \mathrm{p}=0.012)$.

Changes in median ( $\mathrm{min}, \max$ ) of SF-36 subscale scores (quality of life measure) over time are shown in Table 3. Results showed that there were no significant changes over time in any of the subscale scores $(\mathrm{p}>0.05)$.

\begin{tabular}{|c|c|c|}
\hline \multirow{8}{*}{$\begin{array}{l}\text { Table 1: } \\
\text { Frequency (\%) } \\
\text { of Participant } \\
\text { Demographics } \\
(n=16)\end{array}$} & \multirow{2}{*}{$\begin{array}{l}\text { Gender } \\
\text { Male }\end{array}$} & \multirow[b]{2}{*}{$9(56.3)$} \\
\hline & & \\
\hline & Activity Level & \\
\hline & $\begin{array}{r}\text { Moderate } \\
\text { Light }\end{array}$ & $\begin{array}{l}15(93.8) \\
1(6.2)\end{array}$ \\
\hline & Work Shift & \\
\hline & $\begin{array}{r}\text { Day } \\
\text { Night }\end{array}$ & $\begin{array}{l}15(93.8) \\
1(6.2)\end{array}$ \\
\hline & Occupation & \\
\hline & $\begin{array}{r}\text { Graduate Student } \\
\text { Healthcare } \\
\text { Mechanic }\end{array}$ & $\begin{array}{l}11(68.8) \\
4(25.0) \\
1(6.2)\end{array}$ \\
\hline
\end{tabular}


Table 2: Mean (SE) of the outcome variables over time

\begin{tabular}{|c|c|c|c|c|}
\hline & Baseline & 4 weeks & $\begin{array}{l}\text { Mean Difference* } \\
(95 \% \mathrm{Cl})\end{array}$ & p-value ${ }^{* * *}(d)$ \\
\hline Stress & $14.1(1.9)$ & $11.0(1.4)$ & $3.1(-0.2,6.4)$ & $0.03^{* *}(0.50)$ \\
\hline Sleep & $5.5(0.8)$ & $4.6(0.5)$ & $0.9(-0.2,1.9)$ & $0.30(0.27)$ \\
\hline Weight (kg) & $80.0(4.4)$ & $79.1(4.6)$ & $0.9(-0.2,1.9)$ & $0.10(0.44)$ \\
\hline BMI $\left(\mathrm{kg} / \mathrm{m}^{2}\right)$ & $27.4(1.3)$ & $27.0(1.3)$ & $0.4(0.0,0.7)$ & $0.06(0.50)$ \\
\hline Body Fat (\%) & $26.2(2.4)$ & $25.5(2.6)$ & $0.7(0.2,1.2)$ & $0.01(0.68)$ \\
\hline $\begin{array}{l}\text { Body Fat Mass } \\
\text { (kg) }\end{array}$ & $21.6(3.0)$ & $21.0(3.2)$ & $0.6(0.1,1.1)$ & $0.02(0.58)$ \\
\hline $\begin{array}{l}\text { Visceral Fat } \\
\left(\mathrm{cm}^{2}\right)\end{array}$ & $98.2(15.3)$ & 94.1 (15.7) & $4.1(1.6,6.6)$ & $0.003(0.81)$ \\
\hline SMM (kg) & $33.0(1.8)$ & $32.8(1.8)$ & $0.2(-0.3,0.6)$ & $0.52(0.22)$ \\
\hline $\begin{array}{l}\text { Lean Body Mass } \\
(\mathrm{kg})\end{array}$ & $15.7(0.8)$ & $15.6(0.8)$ & $0.1(-0.1,0.3)$ & $0.59(0.21)$ \\
\hline Trunk Fat (kg) & $11.5(1.6)$ & $11.0(1.6)$ & $0.5(0.2,0.7)$ & $0.001(1.10)$ \\
\hline
\end{tabular}

Abbreviations: SE, Standard Error; Cl, Confidence Interval; BMI, Body Mass Index; SMM, Skeletal Muscle Mass; $d=$ difference in means/standard deviation (Cohen's d)

\section{DISCUSSION}

Based on an extensive literature review, to the best of our knowledge, this is the first study to examine the effects of a 4 week, 16:8 IF / TRF protocol with no required or self-reported caloric deficit in healthy young adults aged $18-65$ years, that evaluated cumulatively stress levels, sleep quality, hunger level, quality of life, and body composition. Results showed significant decreases in stress, body fat \%, body fat mass, visceral fat, trunk fat, and hunger levels. However, there was no significant change in sleep quality or quality of life. Participant hunger levels decreased over the four weeks suggesting acclimation to the IF paradigm.

A strength of our pilot study was participant compliance. Based on participant feedback, participants stated that the IF paradigm was practical. One participant stated, "It was hard at first, but I felt better fast". Another stated, "It helped reset my gut and give it a break from digesting all day". Participants were supplied with a compliance log sheet to monitor daily adherence. This provided participants a route for self-accountability. A second strength was routine communication. This encouraged participants to discuss the difficulties they faced that might have hindered their compliance. In addition, investigators received positive feedback to develop future studies. Further research is needed to expand these positive findings with a larger sample size (of varying ages, gender groups, body habitus, and health conditions), a control group, over different time periods, and with repeat assessments after study completion. Additionally, biomarker assessments of cortisol and melatonin should be performed.

Relationships between stress levels and fasting are based on hormones involved, number of calories consumed, and the duration of the fast. During stress, hormone levels of cortisol, catecholamines, and insulin change [37]. We propose the decrease in stress levels seen in our study participants was possibly due to a decrease in the level of cortisol through the IF / TRE program, possibly mediated through improved overall biomarkers of health as suggested by the optimistic body composition changes. Other sources include confounding variables such as: outside sources such as life events, or even the Hawthorne Effect of being a research participant. In prior trials there has been a strong association between visceral fat and overall body fat percentage with cortisol levels, favoring our first hypothesis [38]. Our protocol duration

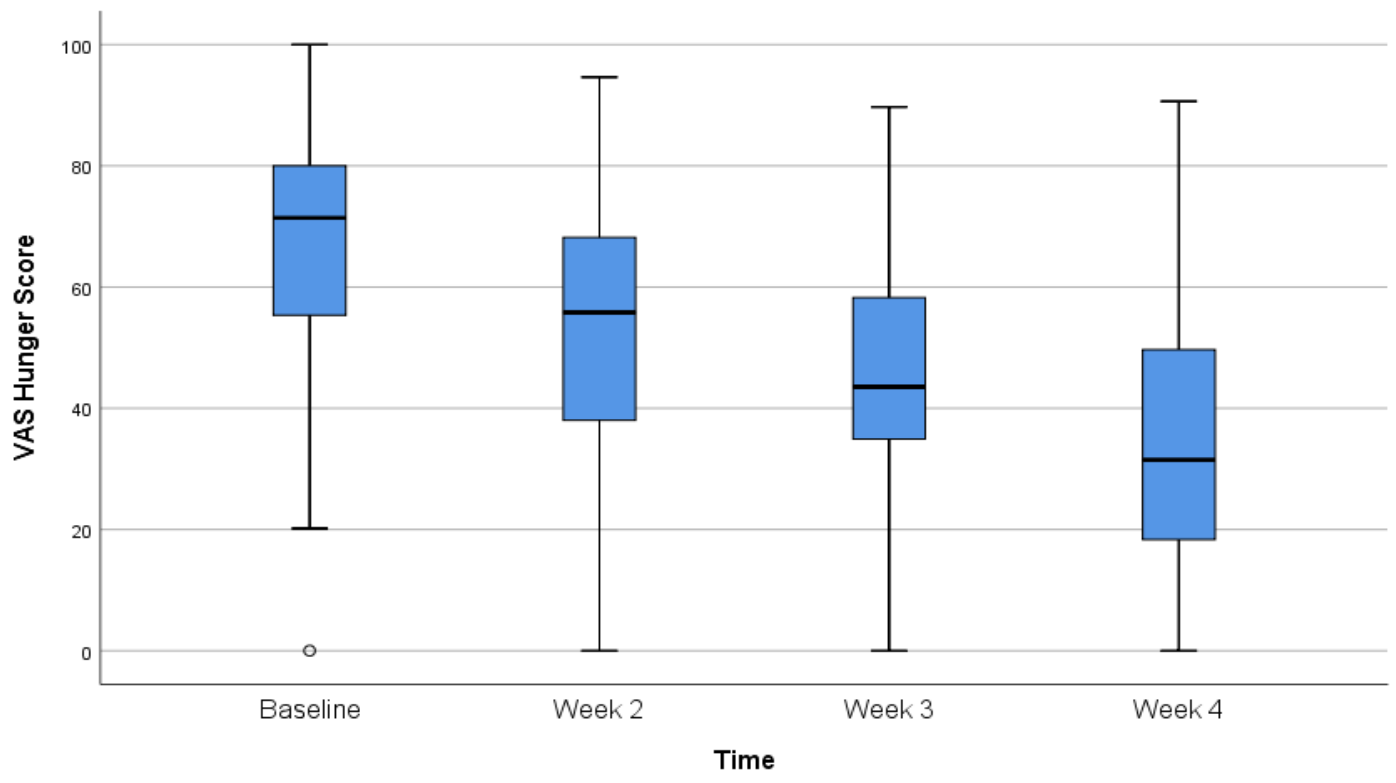

Figure 2. Changes in median (min, max) of VAS hunger scores over time 
Table 3: Median (min, max) of SF-36 subscale scores over time

\begin{tabular}{llll}
\hline & Baseline & 4 weeks & p-value (r) \\
\hline Physical Functioning & $3.0(2.8,3.0)$ & $3.0(2.8,3.0)$ & $0.16(0.35)$ \\
\hline Role Limitations-Physical Health & $2.0(1.0,2.0)$ & $2.0(2.0,2.0)$ & $0.07(0.46)$ \\
Role Limitations-Emotional & $2.0(1.0,2.0)$ & $2.0(1.0,2.0)$ & $0.67(0.11)$ \\
\hline Energy/Fatigue & $3.5(2.5,4.8)$ & $3.8(2.5,4.8)$ & $0.53(0.16)$ \\
Emotional Well-being & $4.2(3.8,5.2)$ & $4.1(3.8,4.8)$ & $1.0(0.00)$ \\
\hline Social Functioning & $3.0(2.5,3.5)$ & $3.0(2.0,3.5)$ & $0.21(0.32)$ \\
Pain & $1.5(1.0,3.0)$ & $1.3(1.0,2.5)$ & $0.23(0.30)$ \\
\hline General Health & $3.0(2.4,3.4)$ & $2.7(2.0,3.2)$ & $0.14(0.37)$ \\
\hline
\end{tabular}

Abbreviation: $r=Z / \sqrt{ } N$

spanned four weeks, however in comparison to another single trial, during an 8 week 16:8 fasting protocol, no change in serum cortisol levels were noted [16].

In the present study, there was preservation of skeletal muscle mass / lean body mass. We suggest our protocol which did not require caloric restriction led to no change in skeletal muscle mass via mechanisms of decreased lean tissue catabolysis. Our results agree with Moro et al. (2016) in which 8 weeks of IF (with no change in caloric intake) showed no change in muscle mass or lean body mass in athletes [16].

Insulin plays a crucial role in substrate utilization for energy. Insulin prevents lipid metabolism in adipose tissue $[39,40]$. While levels of insulin increase due to carbohydrate consumption, they are lowered during fasting. This mechanism suggests a "flipping of the metabolic switch" from carbohydrate / glucose substrate energy utilization to lipid substrate energy utilization $[41,42]$. This leads to an increase in lipid metabolism. We suggest this as a possible mechanism as to why body fat percentage / body fat mass were significantly decreased in the present study.

Many studies investigating sleep architecture and IF have been completed on diurnal fasting during Ramadan. Conversely, few studies have been conducted on the effects of 16:8 IF on sleep architecture specifically. In the present study, there was no significant change in sleep quality during the four weeks of fasting, similar to a prior assessment in individuals with obesity which suggested a lack of sleep disruption caused by 16:8 IF / TRF [27]. More importantly, sleep quality did not worsen. Although not significant, sleep quality trended towards improvement with a $16.4 \%$ enhancement indicating potential clinical importance. Michalsen et al., during a 7 -day fasting program, noticed a significant decrease in sleep arousals and increase in overall sleep quality in non-obese individuals [43] and Queiroz et al. showed improvements in sleep patterns in overweight individuals [44]. The human body functions on circadian clocks. These clocks, through gene expression, communicate the circadian rhythms that lead to overall homeostasis. Fasting and timing of food consumption can alter circadian clocks [45].

Baseline SF-36 Quality of Life levels were at a high for all participants. At the conclusion of the study, Quality of Life maintained high pre-study levels. It is possible decreased stress levels, improvements in body composition, and stabilization of sleep patterns played a role in maintaining a high level of Quality of Life by the completion of the study.

\section{LIMITATIONS}

Due to the small sample size, single location and short study duration of four weeks, our results may not be generalizable. Many participants were associated with a health care university (students, faculty, and staff) and so carried high baseline knowledge of health behaviors and their associated effects. Although participants were expected to maintain their daily caloric intake with no mandated caloric deficit, participants did not track daily calories. Therefore, by having no controlled diet, we are unable to truly infer participants maintained their daily caloric intake. It is possible that caloric intake could have decreased with the time of feeding condensed. Biomarker assessments of cortisol and melatonin were not measured and could, in future investigations, augment self-reported wellness measures. Due to this being a pilot study to demonstrate the feasibility of concept, a control group was not utilized.

\section{CONCLUSION}

Intermittent Fasting in the form of a 16:8 daily Time-Restricted Feeding protocol can potentially be an effective weight management option to help decrease acute stress levels, lower body fat (more importantly visceral fat), decrease hunger (thus increasing satiety), while also not disrupting sleep quality and quality of life. This in turn, could potentially aid in decreasing obesity-related diseases. Our pilot study demonstrated many health related benefits from a TRF program with no prescribed caloric deficit that could easily be incorporated by healthcare workers into personal and patient whole-person wellness programs. Such a program could bolster resilience of healthcare workers and could improve a multitude of health related patient outcomes.

\section{REFERENCES}

1. Organization WH. Obesity and overweight 2020 [Available from: https://www.who.int/news-room/fact-sheets/ detail/obesity-and-overweight

2. Hales C, Carroll M, Fryar C, Ogden C. Prevalence of Obesity and Severe Obesity Among Adults: United States, 2017-2018. In: Control Cf D, editor. Hyattsville (MD): National Center for Health Statistics; 2020.

3. Harvie M, Howell A. Potential Benefits and Harms of Intermittent Energy Restriction and Intermittent Fasting Amongst Obese, Overweight and Normal Weight Subjects-A Narrative Review of Human and Animal Evidence. Behav Sci (Basel). 2017 Jan;7(1):E4.

4. One-Third of Americans Are Dieting, Including One in 10 Who Fast While Consumers Also Hunger for Organic, "Natural" and Sustainable [Internet]. International 
Food and Information Council. 2018. Available from: https://foodinsight.org/one-third-of-americans-are-dieting-including-one-in-10-who-fast-while-consumersalso-hunger-for-organic-natural-and-sustainable/

5. Rynders CA, Thomas EA, Zaman A, Pan Z, Catenacci VA, Melanson EL. Effectiveness of Intermittent Fasting and Time-Restricted Feeding Compared to Continuous Energy Restriction for Weight Loss. Nutrients. 2019 Oct;11(10):E2442.

6. Swift DL, Johannsen NM, Lavie CJ, Earnest CP, Church TS. The role of exercise and physical activity in weight loss and maintenance. Prog Cardiovasc Dis. 2014 Jan-Feb;56(4):441-7.

7. Strychar I. Diet in the management of weight loss. CMAJ. 2006 Jan;174(1):56-63.

8. Ahmed S, Singh D, Khattab S, Babineau J, Kumbhare D. The Effects of Diet on the Proportion of Intramuscular Fat in Human Muscle: A Systematic Review and Meta-analysis. Front Nutr. 2018 Feb;5:7.

9. Benton D, Young HA. Reducing Calorie Intake May Not Help You Lose Body Weight. Perspect Psychol Sci. 2017 Sep;12(5):703-14.

10. Scheen AJ. The future of obesity: new drugs versus lifestyle interventions. Expert Opin Investig Drugs. 2008 Mar;17(3):263-7.

11. Hall KD, Kahan S. Maintenance of Lost Weight and Long-Term Management of Obesity. Med Clin North Am. 2018 Jan;102(1):183-97.

12. Varkevisser RD, van Stralen MM, Kroeze W, Ket JC, Steenhuis IH. Determinants of weight loss maintenance: a systematic review. Obes Rev. 2019 Feb;20(2):171-211.

13. Dong TA, Sandesara PB, Dhindsa DS, Mehta A, Arneson LC, Dollar AL, et al. Intermittent Fasting: A Heart Healthy Dietary Pattern? Am J Med. 2020 Aug;133(8):901-7.

14. Stockman MC, Thomas D, Burke J, Apovian CM. Intermittent Fasting: Is the Wait Worth the Weight? Curr Obes Rep. 2018 Jun;7(2):172-85.

15. Regmi P, Heilbronn LK. Time-Restricted Eating: Benefits, Mechanisms, and Challenges in Translation. iScience. 2020 Jun;23(6):101161.

16. Moro T, Tinsley G, Bianco A, Marcolin G, Pacelli QF, Battaglia G, et al. Effects of eight weeks of time-restricted feeding (16/8) on basal metabolism, maximal strength, body composition, inflammation, and cardiovascular risk factors in resistance-trained males. J Transl Med. 2016 Oct;14(1):290.

17. Gabel K, Hoddy KK, Haggerty N, Song J, Kroeger CM, Trepanowski JF, et al. Effects of 8-hour time restricted feeding on body weight and metabolic disease risk factors in obese adults: A pilot study. Nutr Healthy Aging. 2018 Jun;4(4):345-53.

18. Hutchison AT, Regmi P, Manoogian EN, Fleischer JG, Wittert GA, Panda S, et al. Time-Restricted Feeding Improves Glucose Tolerance in Men at Risk for Type 2 Diabetes: A Randomized Crossover Trial. Obesity (Silver Spring). 2019 May;27(5):724-32.

19. Rothschild J, Hoddy KK, Jambazian P, Varady KA.
Time-restricted feeding and risk of metabolic disease: a review of human and animal studies. Nutr Rev. 2014 May;72(5):308-18.

20. Martin B, Mattson MP, Maudsley S. Caloric restriction and intermittent fasting: two potential diets for successful brain aging. Ageing Res Rev. 2006 Aug;5(3):332-53.

21. Aly SM. Role of intermittent fasting on improving health and reducing diseases. Int J Health Sci (Qassim). 2014 Jul;8(3):V-VI.

22. Marinac CR, Sears DD, Natarajan L, Gallo LC, Breen CI, Patterson RE. Frequency and Circadian Timing of Eating May Influence Biomarkers of Inflammation and Insulin Resistance Associated with Breast Cancer Risk. PLoS One. 2015 Aug;10(8):e0136240.

23. Turner-McGrievy GM, Dunn CG, Wilcox S, Boutté AK, Hutto B, Hoover A, et al. Defining Adherence to Mobile Dietary Self-Monitoring and Assessing Tracking Over Time: Tracking at Least Two Eating Occasions per Day Is Best Marker of Adherence within Two Different Mobile Health Randomized Weight Loss Interventions. J Acad Nutr Diet. 2019 Sep;119(9):1516-24.

24. Jospe MR, Roy M, Brown RC, Haszard JJ, Meredith-Jones K, Fangupo LJ, et al. Intermittent fasting, Paleolithic, or Mediterranean diets in the real world: exploratory secondary analyses of a weight-loss trial that included choice of diet and exercise. Am J Clin Nutr. 2020 Mar;111(3):503-14.

25. Schroder JD, Falqueto H, Mânica A, Zanini D, de Oliveira $\mathrm{T}$, de Sá CA, et al. Effects of time-restricted feeding in weight loss, metabolic syndrome and cardiovascular risk in obese women. J Transl Med. 2021 Jan;19(1):3.

26. Brady AJ, Langton HM, Mulligan M, Egan B. Effects of 8 wk of 16:8 Time-restricted Eating in Male Middleand Long-Distance Runners. Med Sci Sports Exerc. 2021 Mar;53(3):633-42.

27. Gabel K, Hoddy KK, Burgess HJ, Varady KA. Effect of 8-h time-restricted feeding on sleep quality and duration in adults with obesity. Appl Physiol Nutr Metab. 2019 Aug;44(8):903-6.

28. Isenmann E, Dissemond J, Geisler S. The Effects of a Macronutrient-Based Diet and Time-Restricted Feeding (16:8) on Body Composition in Physically Active Individuals-A 14-Week Randomised Controlled Trial. Nutrients. 2021 Sep;13(9):3122.

29. McAllister MJ, Pigg BL, Renteria LI, Waldman HS. Time-restricted feeding improves markers of cardiometabolic health in physically active college-age men: a 4-week randomized pre-post pilot study. Nutr Res. 2020 Mar;75:32-43.

30. Pellegrini M, Cioffi I, Evangelista A, Ponzo V, Goitre I, Ciccone $\mathrm{G}$, et al. Effects of time-restricted feeding on body weight and metabolism. A systematic review and meta-analysis. Rev Endocr Metab Disord. 2020 Mar;21(1):17-33.

31. Ling CH, de Craen AJ, Slagboom PE, Gunn DA, Stokkel MP, Westendorp RG, et al. Accuracy of direct segmental multi-frequency bioimpedance analysis in the assessment of total body and segmental body 
composition in middle-aged adult population. Clin Nutr. 2011 Oct;30(5):610-5.

32. Lee EH. Review of the psychometric evidence of the perceived stress scale. Asian Nurs Res. 2012 Dec;6(4):121-7.

33. Liu X, Zhao Y, Li J, Dai J, Wang X, Wang S. Factor Structure of the 10-Item Perceived Stress Scale and Measurement Invariance Across Genders Among Chinese Adolescents. Front Psychol. 2020 Apr;11:537.

34. Mollayeva T, Thurairajah P, Burton K, Mollayeva S, Shapiro CM, Colantonio A. The Pittsburgh sleep quality index as a screening tool for sleep dysfunction in clinical and non-clinical samples: A systematic review and meta-analysis. Sleep Med Rev. 2016 Feb;25:52-73.

35. McHorney CA, Ware JE Jr, Lu JF, Sherbourne CD. The MOS 36-item Short-Form Health Survey (SF-36): III. Tests of data quality, scaling assumptions, and reliability across diverse patient groups. Med Care. 1994 Jan;32(1):40-66.

36. Stubbs RJ, Hughes DA, Johnstone AM, Rowley E, Reid $\mathrm{C}$, Elia $\mathrm{M}$, et al. The use of visual analogue scales to assess motivation to eat in human subjects: a review of their reliability and validity with an evaluation of new handheld computerized systems for temporal tracking of appetite ratings. Br J Nutr. 2000 Oct;84(4):405-15.

37. Ranabir S, Reetu K. Stress and hormones. Indian J Endocrinol Metab. 2011 Jan;15(1):18-22.

38. Mujica-Parodi LR, Renelique R, Taylor MK. Higher body fat percentage is associated with increased cortisol reactivity and impaired cognitive resilience in response to acute emotional stress. Int J Obes. 2009 Jan;33(1):157-65.
39. Dimitriadis G, Mitrou P, Lambadiari V, Maratou E, Raptis SA. Insulin effects in muscle and adipose tissue. Diabetes Res Clin Pract. 2011 Aug;93 Suppl 1:S52-9.

40. Zhang L, Keung W, Samokhvalov V, Wang W, Lopaschuk GD. Role of fatty acid uptake and fatty acid beta-oxidation in mediating insulin resistance in heart and skeletal muscle. Biochim Biophys Acta. 2010 Jan;1801(1):1-22.

41. Anton SD, Moehl K, Donahoo WT, Marosi K, Lee SA, Mainous AG 3rd, et al. Flipping the Metabolic Switch: Understanding and Applying the Health Benefits of Fasting. Obesity (Silver Spring). 2018 Feb;26(2):254-68.

42. de Cabo R, Mattson MP. Effects of Intermittent Fasting on Health, Aging, and Disease. N Engl J Med. 2019 Dec;381(26):2541-51.

43. Michalsen A, Schlegel F, Rodenbeck A, Lüdtke R, Huether G, Teschler H, et al. Effects of short-term modified fasting on sleep patterns and daytime vigilance in non-obese subjects: results of a pilot study. Ann Nutr Metab. 2003;47(5):194-200.

44. Queiroz JD, Macedo RC, Tinsley GM, Reischak-Oliveira A. Time-restricted eating and circadian rhythms: the biological clock is ticking. Crit Rev Food Sci Nutr. 2021;61(17):2863-75.

45. Almeneessier AS, BaHammam AS. How does diurnal intermittent fasting impact sleep, daytime sleepiness, and markers of the biological clock? Current insights. Nat Sci Sleep. 2018 Dec;10:439-52. 